\title{
Transport study in unsaturated porous media by tracer experiment in a dichromatic X-ray experimental device
}

\author{
C. Latrille ${ }^{1}$ and M.C. Néel ${ }^{2}$ \\ ${ }^{1}$ Nuclear Energy Commission, Saclay centre, 91191 Gif-sur-Yvette, France \\ ${ }^{2}$ University of Avignon, Department of Physics, 84000 Avignon, France
}

\begin{abstract}
Estimating contaminant migration in the context of waste disposal and/or environmental remediation of polluted soils requires a complete understanding of the underlying transport processes. In unsaturated porous media, water content is one of the most determining parameters to describe solute migration because it impacts directly on solute pore velocity. However, numerous studies are satisfied with only a global or a partial spatial distribution of water content within the studied porous media. Therefore, distribution of water content in porous media must be precisely achieved to optimize transport processes modeling. Tracer experiments with downward flow were performed on the BEETI experimental device equipped with a sand column. Water content and concentration profiles of tracer (KI) were measured along the column during experiment. The relative dispersion of water content, calculated along the column, gives an idea of influence of this parameter on transport properties. A relationship between pore velocity, Darcy flow velocity and water content is proposed.
\end{abstract}

\section{INTRODUCTION}

The prediction of contaminant migration in the context of waste disposal or environmental remediation of polluted soils requires a complete understanding of the underlying transport processes. It is commonly assumed that in homogeneous saturated porous media, the evolution of a tracer pulse is governed by the advection-dispersion equation (ADE) [1], condensing advection, mechanical dispersion and molecular diffusion mechanisms. In permeable porous media such as soils or sands, which are commonly found in environmental applications, hydrodynamic dispersion always plays a relevant role in determining the fate of transport processes. Moreover, migration in unsaturated soils is frequently due to vertical drainage. Within the porous media, the liquid spreads over the solid surface and forms a continuous and connected liquid, fulfilling partially the porous space. It is through this connected configuration, that capillary flow takes place, described by the Richard's equation [2]. Depending on their sizes, pores are fully or partially filled of water. The adsorbed films in contact with the solid (residual water) poorly contribute to capillary flow, but only the free water easily displaced by drainage. Nevertheless, films may significantly participate in spreading, and give tracer particles opportunities of being arrested.

In this context, dispersion also depends on water content, and the links between tortuosity, pore velocity and dispersivity are not entirely understood [3-5]. Solute dispersion studies in unsaturated porous media suggest that the dispersion coefficient increases with decreasing water content $[3,4,6,7]$. Moreover, dispersion coefficient was found to increase with increasing pore velocity for a fixed saturation [6]. Although numerous experiments have been dedicated to solute transport in unsaturated porous media, only a few focused on the influence of various degrees of saturation and even fewer considered saturation gradients [8].
Column experiments performed on unsaturated porous media have frequently evidenced early arrival and a long effluent tailing which cannot be interpreted by ADE. A physical non-equilibrium model, called the mobileimmobile model (MIM) accounts for such results [9] and is commonly used to describe the evolution of solute concentration in porous media (see Eqs. (1) and (2) below). The MIM model assumes that the liquid phase in soil pores can be partitioned into mobile $\theta_{m}$ (flowing) and immobile $\theta_{\mathrm{im}}$ (residual and free water include in pore isolated from the main flow):

$$
\begin{aligned}
\theta_{m} \frac{\partial C_{m}}{\partial t}+\theta_{i m} \frac{\partial C_{i m}}{\partial t} & =\theta_{m} D_{m} \frac{\partial^{2} C_{m}}{\partial x^{2}}-\theta_{m} v_{m} \frac{\partial C_{m}}{\partial x} \\
\theta_{i m} \frac{\partial C_{i m}}{\partial t} & =\alpha\left(C_{m}-C_{i m}\right) \\
\theta & =\theta_{m}+\theta_{i m} .
\end{aligned}
$$

Here and in the following, $m$ and $i m$ refer to mobile and immobile phases, $\mathrm{C}$ with the concentration $(\mathrm{mol} / \mathrm{L}), \theta$ with the water content (-), $D_{m}=D_{h} /\left(\theta_{m} / \theta\right)$ with $D_{h}$ the hydrodynamic dispersion $\left(D_{h}=d v\right.$ where $v$ is the pore velocity defined by $\left.v=q_{D} / \theta\right), d$ the dispersivity, $v_{m}$ is pore velocity of mobile water $\left(v_{m}=q_{D} / \theta_{m}\right.$, where $q_{\mathrm{D}}$ is the Darcy flow velocity, $v_{m}$ being larger than $v$ ) and $\alpha$ is the first-order mass transfer coefficient between the mobile and immobile water regions. Values of $\theta_{m}$ and $v_{m}$ are assumed constant in space and time.

Water content appears to be one of the most determining parameters to describe solute transport in unsaturated porous media. It impacts directly on pore velocity which determines the arrival time and, to a lower extent, on the dispersion coefficient. Most of the laboratory experiments performed on columns containing porous media saturated with uniform water profile, reports a decrease of mobile water fraction $\left(\theta_{m} / \theta\right)$ as the water content decreases $[4,10,11]$. Water content is 
usually estimated by weighing the column [7,12] or with some local probes incorporated within the porous media, measuring water content or water pressure [35]. When probes were used to measure the water content, interpolation and extrapolation were performed to simulate water distribution along the column. Water content fluctuations and uncertainties of water content measurements are not always taken into account when determining transport parameters and simulating solute transport. Only few researches, in this context, have access to a precise water content distribution $[11,13$, 14]. Diffusion in immobile water is usually considered as the dominant mechanism to explain the appearance of asymmetric and long-tailed breakthrough curves in unsaturated and/or double porosity media. The reciprocal influences of various transport mechanisms depend on the flow. In saturated media, it is resumed by pore velocity defined as Darcy flow velocity divided by water content $\left(v=q_{D} / \theta\right)$. A similar expression is often assumed in unsaturated conditions, within the context of single (ADE) or dual porosity (MIM) models. In most cases, water content and pore velocity are not directly measured within the porous media during the experiment. Hypotheses on uniformity of water distribution and constant pore velocity are formulated, but not verified.

The objectives of this study are (1) to investigate the impact of water content accuracy on the transport parameters determination in a porous media with saturation gradient and (2) to verify the link between pore velocity, water content and Darcy flow velocity. An experimental set-up was specifically conceived to assess the spatial and temporal dynamics of tracers in relation to the geometry and the physico-chemical conditions of porous media. Spatial water distribution and tracer concentration were simultaneously measured within a column filled of porous media in which hydrodynamic conditions were controlled.

\section{MATERIAL AND METHODS}

\subsection{Transport experiments}

\subsubsection{Experimental device and operating conditions}

The BEETI experimental device [13] is composed of a vertical polycarbonate column filled of porous media, a controlled hydraulic circuit that enables tracer migration within the column, and a dichromatic X-ray spectrometer (DXS) for measuring the porous media properties (porosity, bulk density and water saturation) and tracer concentrations within the column (Fig. 1). This technique is non-intrusive and non-destructive. Experiments with downward flow are performed by injecting background and tracer solutions from the upper entrance. The solutions are drained by a pump, which regulates the solution flow rate and the tracer injection at the column inlet. The steady state Darcy flow velocity $\mathrm{q}_{\mathrm{D}}$ is also verified by weighing the outgoing solutions. The pressure head (h) is imposed by adjusting the position of the outflow bottle. The unsaturated condition is maintained by suction at the bottom of the column $\mathrm{h}=0 \mathrm{~cm}$ and the constant

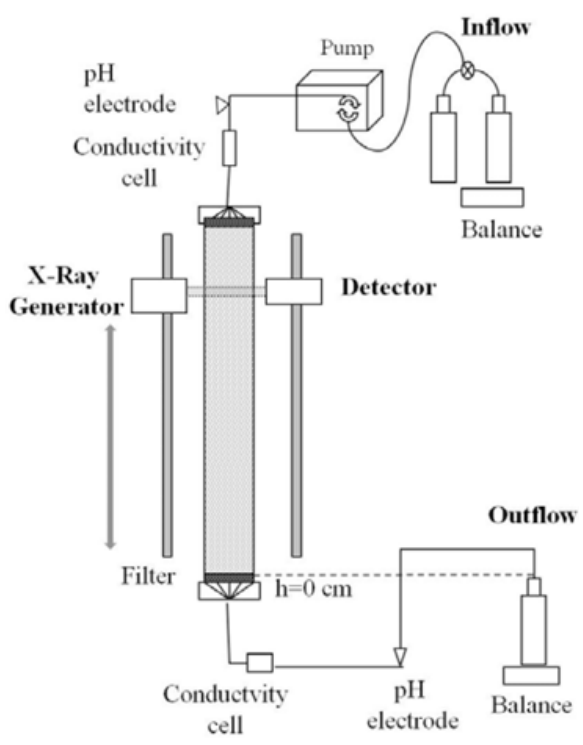

Figure 1. The BEETI experimental set-up.

flow rate at the upper entrance. Tracer experiments are only performed when physical equilibrium water pressure due to suction and solution flow is reached and experimentally checked by water content and Darcy flow velocity measuring. Electrical conductivity cells and $\mathrm{pH}$ electrodes are placed on line at the column inlet and outlet, to measure the tracer displacement through the column and the physico-chemical conditions. At the outlet of the column, the concentration profile coincides with the breakthrough curve, which is the most frequently measured quantity in contaminant migration experiments and provides information about the tracer mean displacement over the entire column length.

The column has $82 \mathrm{~cm}$ height and internal diameter of $5 \mathrm{~cm}$. The column is filled with homogeneously mixed Fontainebleau quartz sand, with bulk density of $1.77 \pm$ $0.01 \mathrm{~g} / \mathrm{cm}^{3}$ and average grain diameter of $200 \mu \mathrm{m}$. The sand is previously washed in pure water (MilliQ) and equilibrated with a $10^{-3} \mathrm{M} \mathrm{KCl}$ solution, to minimize the ionic exchange between solid sites surface and tracer. In order to obtain a homogeneously packed porous medium, the dry sand was poured continuously and packed while vibrating the column. At first, sand column saturation is imposed by gradually injecting a $\mathrm{KCl}$ solution, at the column bottom. Grains stacking homogeneity and saturation of the medium are measured by DXS, which allows estimating bulk density and porosity along the column with a resolution of $10 \mathrm{~mm}$ in the longitudinal direction. A resulting estimate of the overall average porosity was $\varepsilon=0.333 \pm 0.050(1 \sigma)$, to be compared with the value $\varepsilon=0.328$ as obtained by weighing the sand poured into the column. All measurements were performed at constant room temperature $\mathrm{T}=20^{\circ} \mathrm{C}$. The background saturating solution contains $\mathrm{KCl}$ at a molar concentration of $10^{-3} \mathrm{~mol} / \mathrm{L}$ (molar mass equal to $74.5 \mathrm{~g} / \mathrm{mol}$ ). The injected tracer is KI (molar mass equal to $166 \mathrm{~g} / \mathrm{mol}$ ), at a molar concentration of $0.11 \mathrm{~mol} / \mathrm{L}$. No density effect on the flow can be evidenced at these concentration and flow conditions. The Darcy flow velocity was $\mathrm{q}_{\mathrm{D}}=2 \mathrm{~cm} / \mathrm{h}$ 
and the duration of the tracer injection is $\tau=2.5 \mathrm{~h}$. Three experimental runs permit to measure the tracer displacement at the points located at distances $\mathrm{x}=12$, $20,22,28,36,40,46,49,52,56,58,60,62,70,78 \mathrm{~cm}$ from the column inlet. Measurement points thus cover the entire retention curve resulting from the above hydraulic conditions. In sake of simplicity, only 6 of them are presented in this work.

\subsubsection{Dichromatic $X$-Ray treatment}

The dichromatic X-ray spectrometer (DXS) combines an $\mathrm{X}$-ray generator and a NaI detector. X-ray beam emitted by a tungsten source is filtrated by a neodymium window that selects the two energy ranges $20-40 \mathrm{keV}$ and $50-$ $75 \mathrm{keV}$, respectively. After crossing the column of porous media, the beam is measured by the NaI detector. Both source and detector are moved along the column by a controlled rack rail and kept in position at specified spatial locations during $60 \mathrm{~s}$ counting time. The X-ray counting measured by the detector depends on the thickness and the nature of the traversed phases (fluid, porous material and tracer) and the counting time. The thickness of each phase is determined by solving the equation given by BeerLambert's law (Eq. (4)):

$$
R=R_{0} \exp \left(-\sum_{i} \mu_{i} \rho_{i} z_{i}\right) .
$$

Here $\mathrm{R}_{0}$ and $\mathrm{R}$ denote respectively the count-rate emitted by the source and the count-rate transmitted throughout the column, detected per unit time for a given X-ray energy range; $i$ is the index of each phase contained in the medium (sand, water, solute tracer); $\mu_{i}\left(\mathrm{~m}^{2} / \mathrm{kg}\right)$ is the mass attenuation coefficient, which depends on the phase composition and on the X-ray energy; $\rho_{i}\left[\mathrm{~kg} / \mathrm{m}^{3}\right]$ is the density, and $\mathrm{z}_{i}(\mathrm{~m})$ the thickness of the phase. Low energy attenuated X-rays are usually overestimated due to the beam hardening and the detector efficiency. Mass attenuation coefficient of each phase is therefore determined in this configuration, taking into account these constrains. Specific standards are used to determine mass attenuation coefficients of sand, $\mathrm{KCl}$ and $\mathrm{KI}$ solutions. Length and composition of these standards are perfectly known and measurable and reproduce the same attenuation coefficients as those of sand and $\mathrm{KCl}$ solution. One of them is selected as reference for the intensity of incident $\mathrm{X}$-rays, $\mathrm{R}_{0}$, since this quantity is not measurable directly, due to saturation phenomena in the detector. Sequences of calibration are performed before the experiments. The $\mu_{i}$ parameters of sand and $\mathrm{KCl}$ solution for each energy range are determined by calibrating the DXS (Table 1). As a result, $6 \mu_{i}$ parameters are determined for sand and $\mathrm{KCl}$ solution. Two molar attenuation coefficients of $\mathrm{KI}$ solution are determined by linear regression of calibration curves obtained with the standard of reference to integrate matrix effect and increasing concentrations. Concentration of $\mathrm{KI}$ is determined by conversion of the corrected counting of the low energy X-Rays range using the equation (4). Differently from [15], mass attenuation
Table 1. Mass attenuation coefficient of $\mathrm{KCl}$ solution and sand solution and molar attenuation coefficient $\left(\mathrm{cm}^{2} / \mathrm{mol}\right)$ of $\mathrm{KI}$ solution obtained by the BEETI experimental device.

\begin{tabular}{ccc}
\hline & $50-75 \mathrm{keV}$ & $20-40 \mathrm{keV}$ \\
\hline KI & 0.507 & 2.615 \\
\hline \multirow{2}{*}{ KCI } & 0.2057 & 0.2727 \\
& 0.2033 & \\
\hline \multirow{2}{*}{ sand } & 0.223 & \multirow{2}{*}{0.4564} \\
& 0.2221 & \\
\hline
\end{tabular}

coefficient is considered invariable, as being specific of each phase. Moreover, these experimental chemical conditions preserve on chemical sorption of KI on the sand surface what confers a conservative behaviour to him. Consequently, KI reflects water flow in these porous media type. At each location within the column, measurements are repeated with a frequency of some minutes. Each measurement integrates the tracer concentration evolution and quantifies its migration through the porous material.

\subsection{Transport parameter determination}

The method of temporal moments for concentration distribution is used to determine the tracer pore velocity from the concentration profiles at the selected location within the column. The $n$-th temporal moment of a concentration profile at a location $x$ is defined as

$$
M_{n}(x)=\int_{0}^{\infty} t^{n} c(x, t) d t .
$$

After normalization we obtain

$$
m_{n}(x)=\frac{M_{n}(x)}{M_{0}(x)}=\frac{\int_{0}^{\infty} t^{n} c(x, t) d t}{\int_{0}^{\infty} c(x, t) d t} .
$$

Here the 0-th order moment simply represents mass conservation during transport, which is a reasonable hypothesis, since for our experimental conditions the tracers are non-reactive. The first normalized moment gives the average residence time of the tracer molecules within the porous medium. The average pore velocity of tracer between the inlet and position $\mathrm{x}$ is thus given by

$$
v(x)=\frac{x}{m_{1}(x)-\frac{\tau}{2}} .
$$

The tracer pore velocity sets equal to pore velocity (pore velocity of $\mathrm{KCl}$ solution) because it is assumed that no chemical retention occurs. The tracer pore velocity deduced from equation (7) is compared with the theoretical pore velocity of the $\mathrm{KCl}$ solution defined by $\mathrm{v}_{(\mathrm{x})}=\mathrm{q}_{\mathrm{D}} / \theta_{(\mathrm{x})}$; $\mathrm{q}_{\mathrm{D}}$ being the Darcy flow velocity (measured by weighing the outlet solution) and $\theta_{(\mathrm{x})}$, the average water content between 0 and $x$. Effectively, at each selected depth $\mathrm{x}$, the measured concentration profile results from all previous tracer history between the inlet at $\mathrm{x}=0$ and $\mathrm{x}$, the depth of 
measurement. When a water content gradient is associated with a steady Darcy flow velocity, pore velocity changes with the water content and cannot be assumed constant all along the column. Moreover, tracer residence time deduced from temporal moments method, comprises time resulting from advection-dispersion processes and time due to transfer between mobile and immobile fractions. The total water content $\left(\theta=\theta_{\mathrm{im}}+\theta_{\mathrm{m}}\right)$ is then included in the tracer concentration profile. The tracer pore velocity must be compared with the $\mathrm{KCl}$ solution pore velocity itself deduced from the total water content, averaged between $\mathrm{x}=0$ and $\mathrm{x}$.

Transport parameters are determined by inverse modelling techniques using the HYDRUS-1D finite element code [16]. The code solves numerically Richard's equation [2] for water flow parameters $K(\theta)$ and $h(\theta)$ determined on experimental retention curves using the van Genuchten - Mualem expression [1]. Then it solves the MIM solute transport equation [9]. The code uses the Levenberg-Marquardt algorithm [17] for parameter optimization by inverse modelling. Due to tailing on concentration profiles, a MIM model is used to determine transport parameters. The input parameters are measured $\mathrm{q}_{\mathrm{D}}$, water content profiles described by van Genuchten - Mualem parameters, tracer concentration and injection time. Flux concentration (Cauchy condition) is imposed as inlet conditions and a zero concentration gradient at infinity as outlet condition. Darcy flow velocity is constant all along the column. Transport parameters $\left(d, \alpha, \theta_{\text {im }}\right.$ and v) are determined at each selected locations for two retention curves, describing the water content profile of the mean water content values $\left(\theta_{\text {mean }}\right)$ and of the minima water content values $\left(\theta_{\min }\right)$ measured along the column.

\section{RESULTS AND DISCUSSION}

\subsection{Water content distribution within the porous media}

Water retentions curves are measured at each centimetre, using the BEETI device, before and after each tracing experiment, representing 11 replicates (Fig. 2).

Measurements are performed on the first $78 \mathrm{~cm}$ of the column. These curves are close, hence difficult to distinguish. Between the top and a depth of $40 \mathrm{~cm}$, water content is nearly constant around 0.18 . Between 40 and $78 \mathrm{~cm}$ depth, a capillary fringe lets water content progressively increase to reach full saturation value at the bottom. Retention curves record local fluctuations of water contents that cannot be related to sand grain stacking, seeing the sand bulk density distribution measured by DXS. These fluctuations may be due to local variations of water repartition, which continue during draining and tracer experiments (Fig. 3). No noticeable systematic increase of water content, associated to successive experiments, was observed during two months.

Relative standard deviations of water content defined as the standard deviation divided by the average value are determined with a $1 \mathrm{~cm}$ step (Fig. 4). It expresses the relative dispersion of water content values around the mean value, due to uncertainty of measurement. Uncertainties

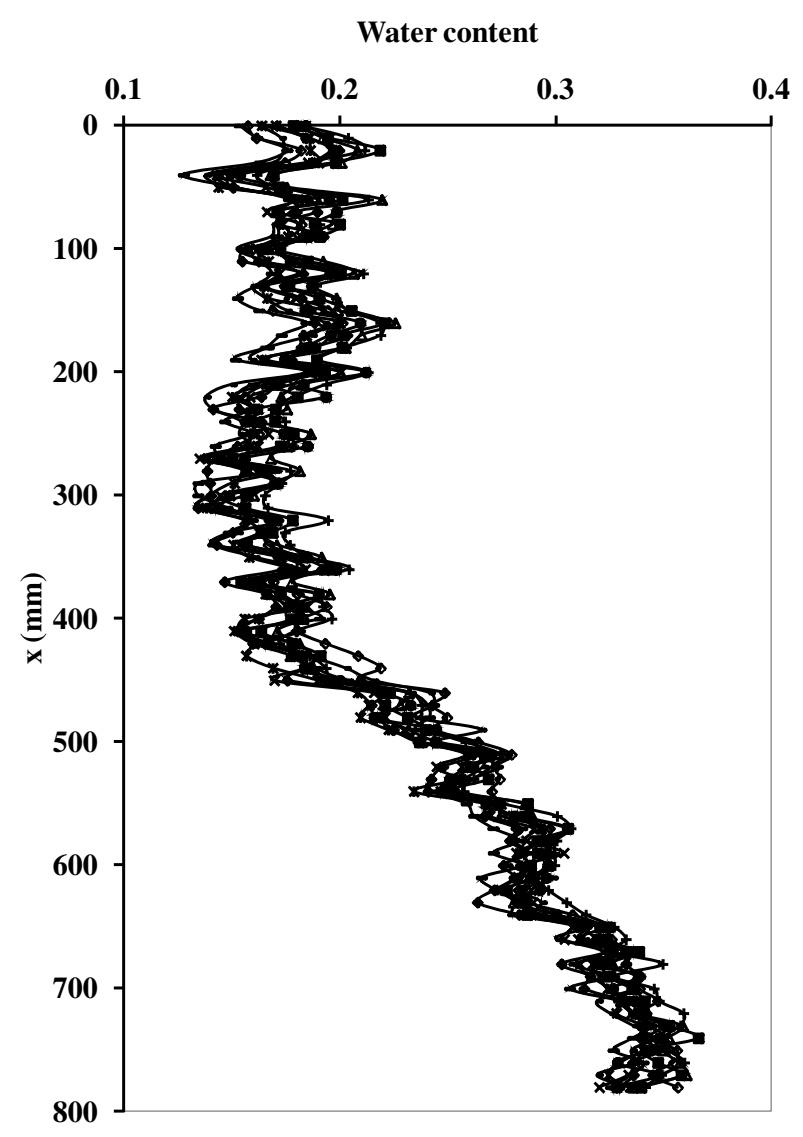

Figure 2. Water content distribution along the unsaturated sand column. 11 replicates.

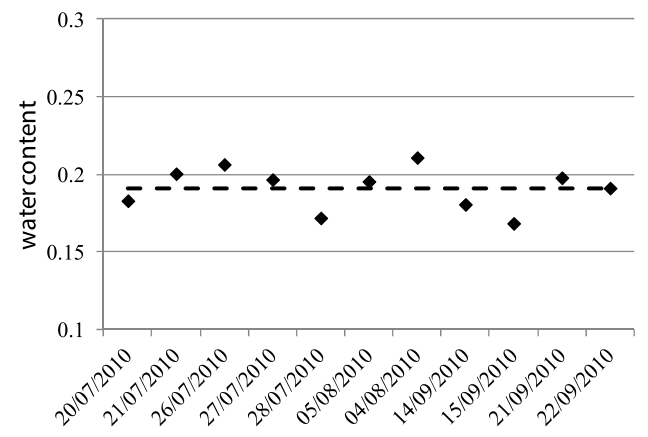

Figure 3. Evolution of the water saturation at location $\mathrm{x}=$ $120 \mathrm{~mm}$, vs. time, compared with the mean value (dashed line).

have larger extent for the lower values of water content ( $3 \%$ to $9 \%$ ) than close to saturation $(1.5 \%$ to $4 \%)$.

Therefore, it appears that such fluctuations including water content uncertainties impact on the water content values describing the global retention curve (Fig. 5). The van Genuchten expression (Eq. (8)) ignores the fluctuations observed on experimental retention curves. Consequently, they are not taking into account in the transport simulations performed with codes using equation (8) (Fig. 5).

From these 11 retentions curves, minima, mean and maxima values of water content are extracted to form probable ranges of the local water content (Fig. 5). Sand-water retention curves are analysed using the 


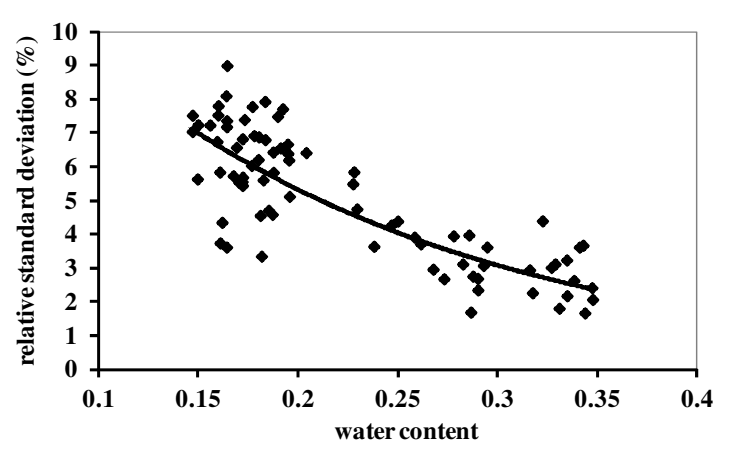

Figure 4. Relative standard deviation of water content at each location, calculated with the 11 replicates, vs. water content.

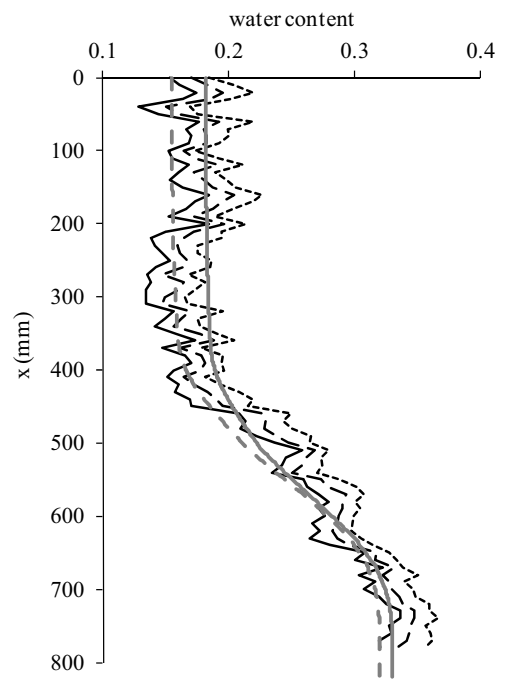

Figure 5. Water content values distribution along the sand column. Minimal values $\theta_{\min }$ (solid line), Mean values $\theta_{\text {mean }}$ (dashed line) and maximal values $\mathrm{m}_{a x i}$ (dotted line). Retention curves from $\theta_{\text {mean }}$ (grey line) and $\theta_{\min }$ (dashed grey line), obtained with van Genuchten parameter in HYDRUS-1D code.

parametric model of [1],

$$
S_{w}=\frac{\theta-\theta_{r}}{\theta_{\text {sat }}-\theta_{r}}=\left(1+|\phi h|^{n}\right)^{-m} .
$$

Where $h$ is the sand-water tension, $n$ and $\phi$ are shape parameters related to the pore size distribution, $m=$ $1-1 / n, \theta_{r}$ is the residual water content retained at solid surface, $\theta_{\text {sat }}(\approx \varepsilon)$ is the saturated water content, $\theta$ is the measured water content and $S_{w}$ is the water saturation. The measured water retention curves for the draining conditions are presented for local water values $\theta_{\max }, \theta_{\text {mean }}$ and $\theta_{\min }$ in Figure 5.

Van Genuchten parameters obtained from $\theta_{\text {mean }}$ and $\theta_{\min }$ retention curves (Table 2) are close but enough different to describe two distinct curves (Fig. 5). Retention curves simulated by HYDRUS-1D code using these parameters are distinct in the upper part of the curves but similar in the lower part.
Table 2. vanGenuchten parameters obtained from $\theta_{\text {mean }}$ and $\theta_{\text {min }}$ retention curves.

\begin{tabular}{ccccc}
\hline & $\theta_{\mathrm{r}}$ & $\theta_{\text {sat }}$ & $\phi$ & $n$ \\
\hline$\theta_{\text {mean }}$ & 0.08 & 0.33 & 0.033 & 3.98 \\
\hline$\theta_{\text {min }}$ & 0.07 & 0.32 & 0.034 & 4.2 \\
\hline
\end{tabular}

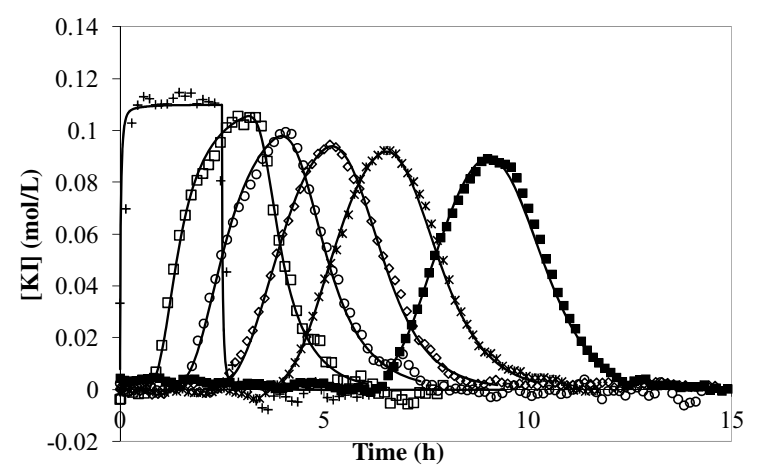

Figure 6. Concentration profiles at various locations from the top column: $0 \mathrm{~cm}$ (cross), $22 \mathrm{~cm}$ (empty square), $40 \mathrm{~cm}$ (circle), $52 \mathrm{~cm}$ (diamond), $62 \mathrm{~cm}$ (star) and $78 \mathrm{~cm}$ (filled square). HYDRUS $1 \mathrm{D}$ fits performed with $\theta_{\text {mean }}$ (line) and $\mathrm{q}_{\mathrm{D}}=\mathrm{q}_{\mathrm{DKI}}=$ $\mathrm{v}_{\mathrm{KI}} \theta_{\text {mean }}$.

\subsection{Impact of water content value on transport parameters determination}

Tracer concentrations are locally measured at selected locations, each of them resulting in a concentration profile with respect to time (Fig. 6). These concentration profiles show a tailing, more pronounced on the two first concentration profiles $(22 \mathrm{~cm}$ and $40 \mathrm{~cm})$, i.e. in the most unsaturated part of the column. There, using a mobile-immobile model seems better adapted to determine transport parameters.

The first step consists in taking advantage of measured parameters to reduce the number of parameters to fit. In the first approach, the $\theta_{\text {mean }}$ retention parameters (Table 2) and the Darcy flow velocity $\mathrm{q}_{\mathrm{D}}=2.15 \mathrm{~cm} / \mathrm{h}$ are introduced into HYDRUS-1D. However, it is impossible to reach a good agreement between experimental data and model (Fig. 7). Simulated profiles are found retarded compared with observed profiles.

Local water content variability is therefore tested by simulating the concentration profile, using unchanged Darcy flow velocity, this time with the $\theta_{\min }$ retention parameters (Table 2). A good agreement is obtained (Fig. 7) which shows that water content is a significant parameter. In most transport studies, water contents are not accurately determined; this leads to considering pore velocity (or Darcy flow velocity) as an adjusting variable. Simulations are performed on the basis of $\theta_{\text {mean }}$ retention parameters, upon considering the Darcy flow velocity as a free variable (Fig. 8). Agreement between fits and experimental data is significantly improved.

Transport parameters values deduced from inverse modelling can be said physically relevant only simulated and experiment profile concentration have similar time scale, i.e. they must have the same residence time. Consequently, water content and Darcy flow velocity 


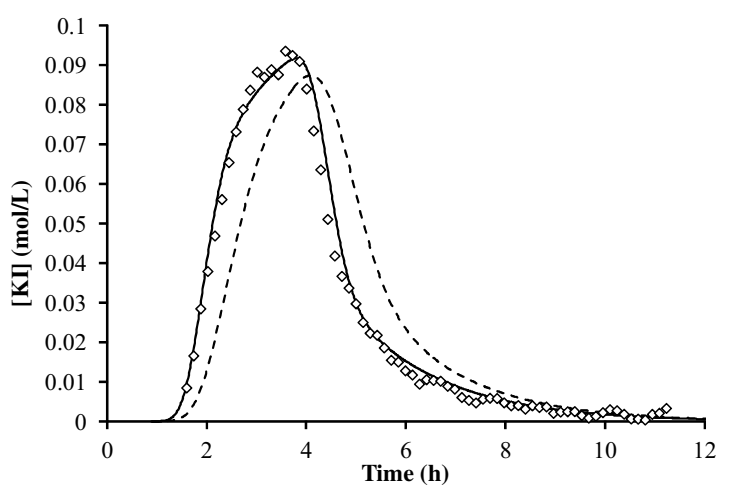

Figure 7. Concentration profile at $36 \mathrm{~cm}$ from the top column (white square). HYDRUS-1D fits performed with $\theta_{\text {mean }}$ (dashed line) and $\theta_{\min }$ (solid line) at flow velocity $\mathrm{q}_{\mathrm{D}}=2.15 \mathrm{~cm} / \mathrm{h}$. Dispersivity $\mathrm{d}=0.8 \mathrm{~cm}$, mass transfer coefficient $\alpha=0.024 \mathrm{~h}^{-1}$ and $\theta_{\mathrm{im}}=0.0422$ are determined with the best fit.

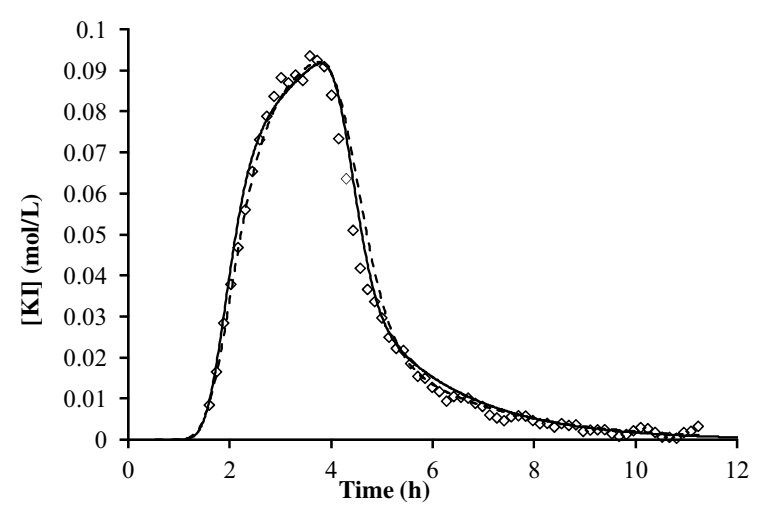

Figure 8. Concentration profile at $36 \mathrm{~cm}$ from the top column (white square). HYDRUS-1D fits performed with $\theta_{\text {mean }}$ and $\mathrm{q}_{\mathrm{D}}=$ $2.41 \mathrm{~cm} / \mathrm{h}$ (dashed line), with $\theta_{\min }$ and $\mathrm{q}_{\mathrm{D}}=2.15 \mathrm{~cm} / \mathrm{h}$ (solid line) at same dispersivity $\mathrm{d}=0.8 \mathrm{~cm}$, mass transfer coefficient $\alpha=0.024 \mathrm{~h}^{-1}$ and $\theta_{\mathrm{im}}=0.0422$.

values play an important role in transport parameters determination.

The transport parameter values deduced from simulation conditions considering pore velocity, dispersivity, immobile water and mass transfert coefficient as adjusting variable and using $\theta_{\text {mean }}$ and $\theta_{\text {min }}$ retention curves are presented in Table 3.

All observations performed in the capillary fringe or saturated zone, show that the dispersion coefficient $(=d v)$ decreases when $\theta_{\text {mean }}$ is increased. Dispersivity, pore velocity and immobile water values increase with decreasing water content when $\theta_{\text {mean }}$ retention curve is used. Differently, these parameters do not significantly vary with water content, if $\theta_{\min }$ retention curve is used. Mass transfer coefficients are larger with $\theta_{\text {min }}$ than $\theta_{\text {mean }}$ retention curve. Relative error achieved for parameter values on the basis of $\theta_{\text {min }}$ related to $\theta_{\text {mean }}$, are ranged between $11 \%$ to $47 \%$ for $\mathrm{d}, 0.2 \%$ to $11 \%$ for $\mathrm{v}(\mathrm{x}), 7 \%$ to $160 \%$ for $\theta_{\text {im }}$ and 25 to $102 \%$ for $\alpha$.

Consequently, a slight uncertainty of the water content value ( 3 to $5 \%$ ) may have a large impact on the transport parameters determination.
Table 3. Dispersivity $(d)$ in $\mathrm{cm}$, pore velocity $\left(v_{(x)}\right)$ and tracer pore velocity $\left(v_{K I}\right)$ in $\mathrm{cm} / \mathrm{h}$, immobile water $\left(\theta_{i m}\right)$ and mass transfer coefficient $(\alpha)$ in $\mathrm{h}^{-1}$ determined using $\theta_{\min }$ and $\theta_{\text {mean }}$ retention parameters at 5 locations within the column.

\begin{tabular}{ccccccc}
\hline $\mathrm{x}(\mathrm{cm})$ & $\theta$ & $\mathrm{d}$ & $\mathrm{v}_{(\mathrm{x})}$ & $\mathrm{v}_{\mathrm{KI}}$ & $\theta_{\mathrm{im}}$ & $\alpha$ \\
\hline \multirow{2}{*}{22} & $\theta_{\text {min }}$ & 0.50 & 13.21 & \multirow{2}{*}{13.13} & 0.04 & 0.10 \\
& $\theta_{\text {mean }}$ & 0.56 & 13.49 & & 0.04 & 0.08 \\
\hline \multirow{2}{*}{40} & $\theta_{\text {min }}$ & 0.50 & 13.66 & \multirow{2}{*}{14.16} & 0.03 & 0.06 \\
& $\theta_{\text {mean }}$ & 0.95 & 15.37 & & 0.02 & 0.04 \\
\hline \multirow{2}{*}{52} & $\theta_{\text {min }}$ & 0.45 & 12.84 & \multirow{2}{*}{12.47} & 0.03 & 0.06 \\
& $\theta_{\text {mean }}$ & 0.83 & 13.29 & & 0.03 & 0.01 \\
\hline \multirow{2}{*}{62} & $\theta_{\text {min }}$ & 0.50 & 11.74 & \multirow{2}{*}{11.23} & 0.04 & 0.24 \\
& $\theta_{\text {mean }}$ & 0.42 & 11.71 & & 0.02 & 0.04 \\
\hline \multirow{2}{*}{78} & $\theta_{\text {min }}$ & 0.35 & 10.23 & \multirow{2}{*}{9.78} & 0.04 & 0.45 \\
& $\theta_{\text {mean }}$ & 0.29 & 9.90 & & 0.02 & 0.04 \\
\hline
\end{tabular}

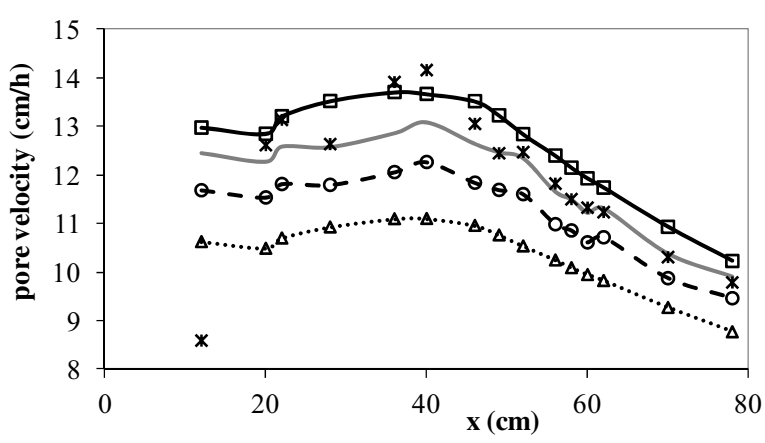

Figure 9. Pore velocity distribution along the sand column. Comparison of pore velocity $\mathrm{v}=\mathrm{q}_{\mathrm{D}} / \theta$ estimated with $\theta_{\min }$ (solid line), $\theta_{\text {mean }}$ (dashed line), $\theta_{\max }$ (dotted line) and $\theta=\theta_{\text {mean }}{ }^{-}$ standard deviation (grey line), with the traceur pore velocity $\mathrm{v}_{\mathrm{KI}}$ (black star).

\subsection{Relationship between pore velocity, water content and flow velocity}

In order to understand the difficulty of fitting the experimental tracer concentration profiles with the MIM model using real flow velocity and mean water content values, the focus turns to the relationship between pore velocity, water content and flow velocity. Tracer pore velocities determined from residence time at each location, using the temporal moment method, are compared with those of the $\mathrm{KCl}$ solution calculated using the measured Darcy flow velocity and the water content values (i.e. $\theta_{\min }, \theta_{\text {mean }}$ and $\left.\theta_{\max }\right)$ averaged between 0 and $\mathrm{x}$. Except the value at the first location, impacted by the entrance, and the values at 36 and $40 \mathrm{~cm}$, all tracer pore velocities are placed between $\mathrm{KCl}$ solution pore velocity curves calculated with $\theta_{\text {mean }}$ and $\theta_{\text {min }}$ values (Fig. 9). Note that tracer pore velocities are very close to those of $\mathrm{KCl}$ calculated with the measured Darcy flow velocity and the $\theta_{\min }$ values. This result indicates that tracer pore velocity is systematically ahead of the pore velocity calculated with the $\theta_{\text {mean }}$ values. However, no chemical retention or anionic exclusion can be suspected in the here addressed porous media, because retention sites have previously been saturated with the $\mathrm{KCl}$ solution. Moreover, no density effect is involved because no asymmetry is observed in the 


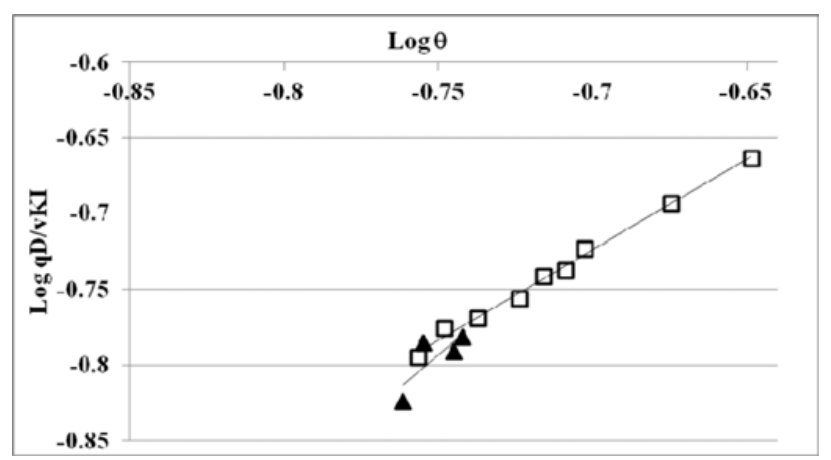

Figure 10. Distribution of tracer pore velocity vs. average water content between 0 and $\mathrm{x}$. Lines are regressions lines for upper points in the column (triangles) and the lower points (square).

leftmost part of the concentration profile [18]. Comparing with $\mathrm{KCl}$ solution pore velocity curves deduced from water contents corresponding to $\theta_{\text {mean }}$ minus the standard deviation calculated on the 11 replicates (Fig. 9), tracer pore velocity indicates two behaviours. In the most unsaturated sand, tracer circulates ahead the $\mathrm{KCl}$ solution whereas tracer and $\mathrm{KCl}$ solution flow simultaneously with the same pore velocity in the capillary fringe until the saturated bottom.

From this we deduce that, tracer pore velocity, Darcy flow velocity and water content satisfy equation (9).

$$
v_{(x)}^{K I}=\frac{b q_{D}}{\theta^{a}}
$$

where the variables $b$ and $a$ are determined by linear regression applied to experimental data (Fig. 10).

Two trends are observed depending on whether measurements are performed (1) in the well and uniform unsaturated media and (2) in the capillary fringe and the saturated media. The linear regression equations are $\mathrm{v}_{\mathrm{KI}}=0.492 \theta^{-1.714}$ (triangles) and $\mathrm{v}_{\mathrm{KI}}=0.178 \theta^{-1.29}$ (square). Knowing $\mathrm{q}_{\mathrm{D}}=2,15 \mathrm{~cm} / \mathrm{h}, b$ is deduced for both equations and is, respectively, 3.10 and 1.51. Values of $a$ are larger than 1 , respectively 1.714 and 1.29 . Tracer behaviour denotes a non-fickian regime mainly in the most unsaturated zone.

\section{CONCLUSIONS}

The mobile-immobile model has been applied successfully to unsaturated transport through homogeneous media [3, $9,10]$. Hence, MIM model is commonly considered valid, at least for well-defined homogeneous unsaturated system. Nevertheless, solute transport experiments performed with BEETI device show that water content, Darcy flow velocity and tracer pore velocity cannot take simultaneously uniform values within a homogeneous packed sand column. Facing difficulties in simulating concentration profiles within the column on the basis of measured Darcy flow velocity and the mean water content, we addressed the impact of water content uncertainty on transport parameter values. It appears that a water content accuracy less than $7 \%$ is not sufficient to determine precisely transport parameters. A new relationship between pore velocity, Darcy flow velocity and water content has been proposed to describe two transport behaviours depending on water content. Further experiments at different flow regimes need being carried out to confirm this last point.

\section{References}

[1] M.T. van Genuchten, Soil Sci. Soc. Am. J. 44, 892 (1980)

[2] L.A. Richards, Physics 1, 318 (1931)

[3] G. Nützmann, S. Maciejewski, K. Joswig, Adv. Water Res. 25, 565 (2002)

[4] I. Padilla, T.-C. J. Yeh, M.H. Conklin, Water Resour. Res. 35, 3303 (1999)

[5] P. Moldrup, T. Olesen, T. Komatsu, P. Schjønning, D.E. Rolston, Soil Sci. Soc. Am. J. 65, 613 (2001)

[6] T. Sato, H. Tanahashi, H.A. Loàiciga, Water Resour. Res. 39, 1155 (2003)

[7] D. Haga, Y. Niibori, T. Chida, Water Resour. Res. 35, 1065 (1999)

[8] A.P. Mortensen, J.W. Hopmans, Y. Mori, J. Simunek, Adv. Water Res. 29, 250 (2006)

[9] M. Th. Van Genuchten, P.J. Wierenga, Soil Sci. Soc. Am. J. 40, 476 (1976)

[10] F. De Smedt, P.J. Wierenga, Water Resour. Res. 20, 225 (1984)

[11] J.P. Gaudet, H. Jégat, G. Vachaud, P.J. Wierenga, Soil Sci. Soc. Am. J. 41, 665 (1977)

[12] J. Lee, D.B. Jaynes, R. Horton, Soil Sci. Soc. Am. J. 64, 492 (2000)

[13] C. Latrille, A. Cartalade, Water-Rock InteractionBirkle and Torres-Alvarado (eds) (Guanajato, 2010)

[14] S. Szenknect, PhD, University of Grenoble (2005)

[15] Šimùnek, J., van Genuchten M. Th. Vadose Zone Journal, Special Issue "Vadose Zone Modeling" 7(2), 587 (2008)

[16] L. Cavé, T. Al, Y. Xiang, P. Vilks, J. Contam. Hydrol. 103, 1 (2009)

[17] More J.J.J. Numer. Anal. 630, 105 (1978)

[18] A. Zoia, C. Latrille, A. Beccantini, A. Cartalade, J. Contam. Hydrol. 109, 14 (2009) 